\title{
Automated Classification of Cerebral Arteries in MRA Images and Its Application to Maximum Intensity Projection
}

\author{
Yoshikazu Uchiyama*, Masashi Yamauchi, Hiromichi Ando, Ryujiro Yokoyama, Takeshi Hara, \\ Hiroshi Fujita, Toru Iwama, and Hiroaki Hoshi
}

\begin{abstract}
Detection of unruptured aneurysms is a major task in magnetic resonance angiography (MRA). However, it is difficult for radiologists to detect small aneurysms on the maximum intensity projection (MIP) images because adjacent vessels may overlap with the aneurysms. Therefore, we proposed a method for making a new MIP image, the SelMIP image, with the interested vessels only, as opposed to all vessels, by manually selecting a cerebral artery from a list of cerebral arteries recognized automatically. By using our new SelMIP viewing technique, the selected vessel regions can also be observed from various directions and would further facilitate the radiologists in detecting small aneurysms. For automated classification of cerebral arteries, two 3D images, a target image and a reference image, are compared. Image registration is performed using the global matching and feature correspondence techniques. Segmentation of vessels in the target image is performed using the thresholding and region growing techniques. The segmented vessel regions were classified into eight cerebral arteries by calculating the Euclidean distance between a voxel in the target image and each of the voxels in the labeled eight vessel regions in the reference image. In applying the automated cerebral arteries recognization algorithm to thirteen MRA studies, results of 10 MRA studies were evaluated as clinically acceptable. Our new viewing technique would be useful in assisting radiologists for detection of aneurysms and for reducing the interpretation time. Keyword: MRA, MIP, Aneurysm
\end{abstract}

\section{INTRODUCTION}

I $\mathrm{N}$ the last decade, approximately 15,000 people died of subarachnoid haemorrhage (SAH) in Japan each year [1]. Most of SAH arise from rupture of aneurysms [2]. Therefore, the detection and management of unruptured aneurysms are an important task for radiologists and/or neurosurgeons. A health check system named Brain Dock has been widely used in Japan for early detection of cerebral and cerebrovascular diseases. Magnetic resonance angiography (MRA), which is considered as a reliable method for early detection of unruptured aneurysms, is routinely employed in the Brain Dock for the screening of aneurysms. However, it is difficult

Manuscript received April 2, 2006. This work was supported in part by a grant for the "Knowledge Cluster Creation Project" from the Ministry of Education, Culture, Sports, Science and Technology, Japan.

*Y. Uchiyama, M. Yamauchi, R. Yokoyama, T. Hara, and H. Fujita are with Dept. of Intelligent Image Information, Graduate School of Medicine, Gifu University, Yanagido 1-1, Gifu-shi, 501-1194, Japan.

H. Ando and T. Iwama are with Dept. of Neurosurgery, Graduate School of Medicine, Gifu University, Japan.

H. Hoshi is with Dept. of Radiology, Graduate School of Medicine, Gifu University, Japan for radiologists to detect small aneurysms in MRA images because of the overlapping of aneurysms and adjacent vessels on maximum intensity projection (MIP) image.

In order to assist radiologists' in interpreting the images, several computer-aided diagnosis (CAD) schemes for detection of aneurysms in MRA images have been developed [3-5]. These CAD schemes can output the locations of candidates of aneurysms by analyzing some image features such as the size and/or the shape of candidates. Radiologists can, then, have a closer examination of the cerebral arteries in the vicinity of the candidates prompted by CAD systems. However, the current CAD systems have a miss rate of about 5\%-10\%. Those missed aneurysms are usually small in size, hence, are very difficult for the radiologists to find. In this paper, we propose a new viewing technique which will help radiologists in detecting small aneurysms. The technique takes on an approach in making a new type of MIP image, called the SelMIP image, with interested vessel only, as opposed to all vessels, by manually selecting a cerebral artery from a list of cerebral arteries. By using our new viewing technique, the selected vessel region can also be observed from various directions, making small aneurysms easy to visualize.

\section{MATERIAL}

Thirteen MRA studies were selected for the evaluation of the proposed method and an additional MRA study was included as the reference. All the MRA images were acquired on a $1.5 \mathrm{~T}$ magnetic image scanner (a Signa Excite Twin Speed 1.5T; GE Medical System, Milwaukee, WI) in the Gifu University Hospital (Gifu, Japan) and were obtained by use of a 3D time-of-flight technique. Each MRA study includes 50 to 140 slice images. The slice images have a fixed size of $256 \times 256$ pixels and the size of the pixels ranges from 0.625 $\mathrm{mm}$ to $0.78 \mathrm{~mm}$. The thickness of each slice is in the range of $0.5 \mathrm{~mm}$ to $1.2 \mathrm{~mm}$. Based on the original MRA slice images, isotropic volume data was generated using linear interpolation. The size of each $3 \mathrm{D}$ volume image was $400 \times 400 \times 200$ voxels and the size of each voxel was $0.5 \times 0.5 \times 0.5 \mathrm{~mm}^{3}$.

\section{Methodology}

\section{A. Overall scheme for automated classification of arteries}

Fig. 1 shows the schematic diagram of the proposed method. 


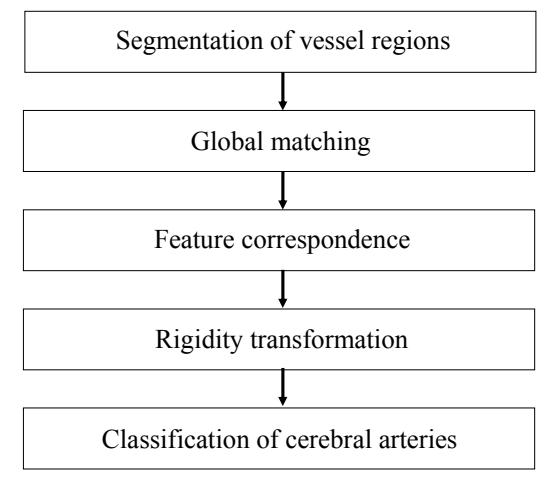

Fig. 1. Schematic diagram of the automated classification of cerebral arteries in MRA images.

The scheme is based on the comparison of the generated 3D volume image of a target MRA study and that of the reference MRA study. In the reference case, eight cerebral arteries were labeled. The eight cerebral arteries are: anterior cerebral artery (ACA), right middle cerebral artery (right MCA), left middle cerebral artery (left MCA), right internal carotid artery right ICA), left internal carotid artery (left ICA), right posterior cerebral artery (right PCA), left posterior cerebral artery (left PCA) and basilar artery (BA). Fig.2 (a) shows a MIP image of the reference MRA study and the eight cerebral arteries were shown and labeled in Fig. 2 (b).

Vessels segmentation and image registration were first performed. Vessels in the 3D target image were segmented using the thresholding and region growing techniques while registration of the $3 \mathrm{D}$ target and reference images was performed using the global matching and feature correspondence techniques. Recognition of each of the cerebral arteries was based on the Euclidean distance measure between the labeled arteries in the $3 \mathrm{D}$ reference image and the segmented vessel voxels in the 3D target image.

\section{B. Segmentation of vessel region}

For segmentation of the vessel regions in the target image, the linear gray-level transform was applied to the 3D MRA image so that the minimum voxel value became zero. Using the cumulative histogram, voxels above the $99 \%$ margin were assigned to have a maximum value of 1024 . After the linear gray-level transformation, vessel regions were segmented from the background by using the gray-level thresholding method with a threshold level of 700 , which was selected empirically. Using this method, large vessels were segmented successfully. However, it is difficult to segment small vessels because the voxel values in small vessel regions were low. Therefore, we used a region growing technique to segment small vessel regions. The segmented large vessel regions were used as "seed" points. Neighboring voxels with values greater than 500 were appended to the seed points.

\section{Global matching}

For global matching, the target image was shifted to align with the reference image based on the segmented vessel

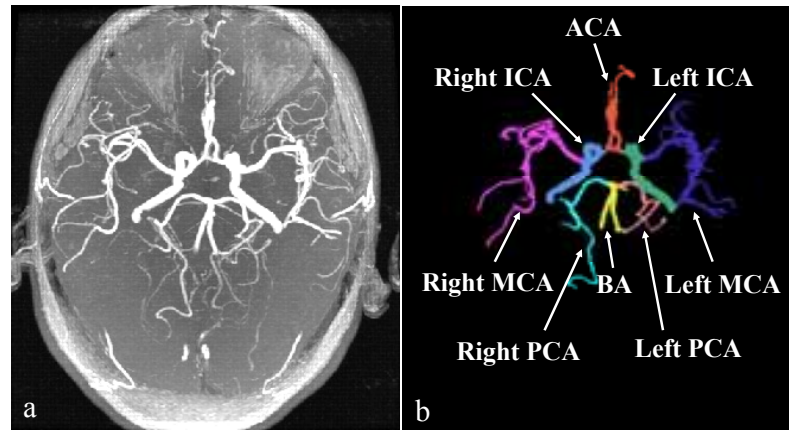

Fig. 2 (a) MIP image of the reference MRA study. (b) Eight cerebral arteries defined by a neuroradiologist. The eight cerebral arteries are labeled and are indicated in different colors.

regions. The translation vector, which corresponds to the shifting of the target image in the $\mathrm{x}, \mathrm{y}$, and $\mathrm{z}$ directions relative to the reference image, was defined so as to maximize the overlapping area of the vessel regions in the target image and the vessel regions in the reference image. Fig. 3 indicates the efficacy of the global matching. Fig.3 (a) shows the superposition of the MIP image of the reference MRA study onto the MIP image of the target MRA study before global matching. Fig.3 (b) shows the result after global matching.

\section{Feature correspondence}

After the global matching, the rigidity transformation was used to achieve a more accurate matching between the 3D target image and the 3D reference image. Template matching was used in determining the coordinates of corresponding points in the rigidity transformation. The similarity measure was determined by the normalized cross-correlation value, $C$, between the reference image $A(i, j, k)$ and the template $B(i, j, k)$ and is given by

$C=\frac{1}{I J K} \sum_{k=1}^{K} \sum_{j=1}^{J} \sum_{i=1}^{I} \frac{\{A(i, j, k)-\bar{a}\}\{B(i, j, k)-\bar{b}\}}{\sigma_{A} \sigma_{B}}$,

where

$\bar{a}=\frac{1}{I J K} \sum_{k=1}^{K} \sum_{j=1}^{J} \sum_{i=1}^{I} A(i, j, k), \quad \bar{b}=\frac{1}{I J K} \sum_{k=1}^{K} \sum_{j=1}^{J} \sum_{i=1}^{I} B(i, j, k)$,

$\sigma_{A}=\sqrt{\frac{\sum_{k=1}^{K} \sum_{j=1}^{J} \sum_{i=1}^{I}(A(i, j, k)-\bar{a})^{2}}{I J K}}$,

$\sigma_{B}=\sqrt{\frac{\sum_{k=1}^{K} \sum_{j=1}^{J} \sum_{i=1}^{I}(B(i, j, k)-\bar{b})^{2}}{I J K}}$

The size of the template $I \times J \times K$ was set to be $21 \times 21 \times 21$. The normalized cross-correlation value indicates the resemblance between the reference and the template. If the images $A$ and $B$ are identical, $C$ will take on the value 1.0. Twelve templates were located manually in the cerebral region of the reference image. Fig.4 (a) shows the central points of twelve templates as white dots. The search area associate with each template in the target image was of size $41 \times 41 \times 41$. A set of coordinates of the corresponding points between the reference and the target image was determined by finding the largest cross-correlation value. Fig.4 (b) shows twelve corresponding points in the target image. 

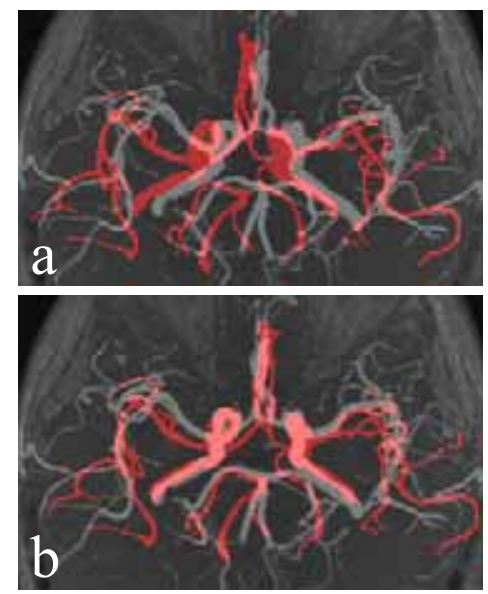

Fig. 3. Illustration of the global matching. The MIP image of a target MRA study (red color) was superimposed onto the MIP image of the reference MRA study (gray color) (a) before (b) after global matching.

\section{E. Rigidity transformation of target image}

By using a set of corresponding control points obtained by the template matching, we determined the translation and rotation vectors between the two images for the rigidity transformation. Let the translation and rotation vectors be denoted by $T$ and $R$, respectively. $P$ and $p$ represent the corresponding points in the reference and the target images, respectively. Assuming the coordinates of the corresponding points in the images after global matching are $\left\{p_{i}=\left(x_{i}, y_{i}, z_{i}\right), P_{i}=\left(X_{i}, Y_{i}, Z_{i}\right): i=1, \ldots, 12\right\}$, the relation between corresponding points in the images can be written as $P_{i}=R p_{i}+T$.

The translation vector $T$ and the rotation vector $R$ can be determined by minimizing

$E^{2}=\sum_{i=1}^{p}\left\|P_{i}-\left(R p_{i}+T\right)\right\|^{2}$.

An efficient algorithm has proposed for determining $T$ and $R$ [7]. In this algorithm, the rotation matrix is obtained first by minimizing

$E_{R}^{2}=\sum_{i=1}^{p}\left\|Q_{i}-R_{i} q_{i}\right\|^{2}$

where $Q_{i}=P_{i}-\bar{P}, q_{i}=p_{i}-\bar{p}$, and $\bar{P}$ and $\bar{p}$ are the centers of gravity of the control points in the reference and target image, respectively. Then, knowing the rotation matrix, the translation vector $T$ is determined from

$$
T=P-R p \text {. }
$$

For calculating the closest corresponding point pairs, we used the iterative closest point (ICP) algorithm [8,9]. The ICP algorithm has two stages and iterations. In the first stage, the set of corresponding points was transformed by using the rigidity transformation of Eq. (2). In the second stage, the closest corresponding point pairs were identified once again. The algorithm terminated when the change in mean square error between iterations fell below a defined threshold.
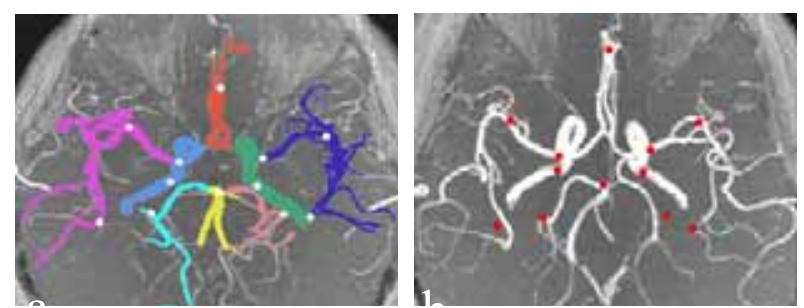

Fig. 4. Corresponding points for the rigidity transformation. (a) The central points of the 12 templates (white dots) projected onto the MIP image of the reference MRA study. (b) Corresponding points (red dots) in the MIP image of the target MRA study.

\section{F. Classification of cerebral arteries}

After the rigidity transformation, all voxels of the segmented vessel regions in the target image were classified into eight cerebral arteries. Classification was based on the Euclidean distance between a voxel $v(x, y, z)$ in the target image and a voxel $a^{i}\left(x^{i}, y^{i}, z^{i}\right),\{i=1, \ldots, 8\}$ in the labeled eight vessel regions in the reference image, i.e.,

$d\left(v, a^{i}\right)=\sqrt{\left(v_{x}-a_{x}^{i}\right)^{2}+\left(v_{y}-a_{y}^{i}\right)^{2}+\left(v_{z}-a_{z}^{i}\right)^{2}}$.

The label yielding the minimum Euclidean distance was considered to be the result of classification. However, a few small regions were not classified correctly, because a part of vessels run in the various directions. Thus, these small regions were classified again into the label surrounding the region, when the size of region was below a defined threshold.

\section{Classification Accuracy of the arteries}

To evaluate the quality of the automated cerebral arteries classification results, subjective rating of the recognized arteries was performed by a Medical Physicist (M.Y.) using a three-point scale as described in the following.

1 (poor): most of the cerebral arteries are not well recognized;

2 (adequate): most of the cerebral arteries are well recognized but with some minor registration errors;

3 (good): all cerebral arteries are perfectly recognized.

The results of the subjective evaluation were that 8 cases were rated 'good', 2 were rated 'adequate', and 3 were rated 'poor'. The results rated 2 or 3 are considered acceptable and would be adequate for clinical use. Overall, the segmentation and classification of cerebral arteries in $76.9 \%(10 / 13)$ of the MRA studies attained a clinically acceptable result. Fig 5 shows example of a 'good' and a 'poor' classification results.

\section{THE SELMIP IMAGE}

After the recognition of individual cerebral arteries in a MRA study, the SelMIP image can be generated. The SelMIP image is a MIP image with only the interested vessels, rather than all vessels, displayed. The interested vessels are manually selected from a list of eight cerebral arteries recognized using the above described procedure. In the 


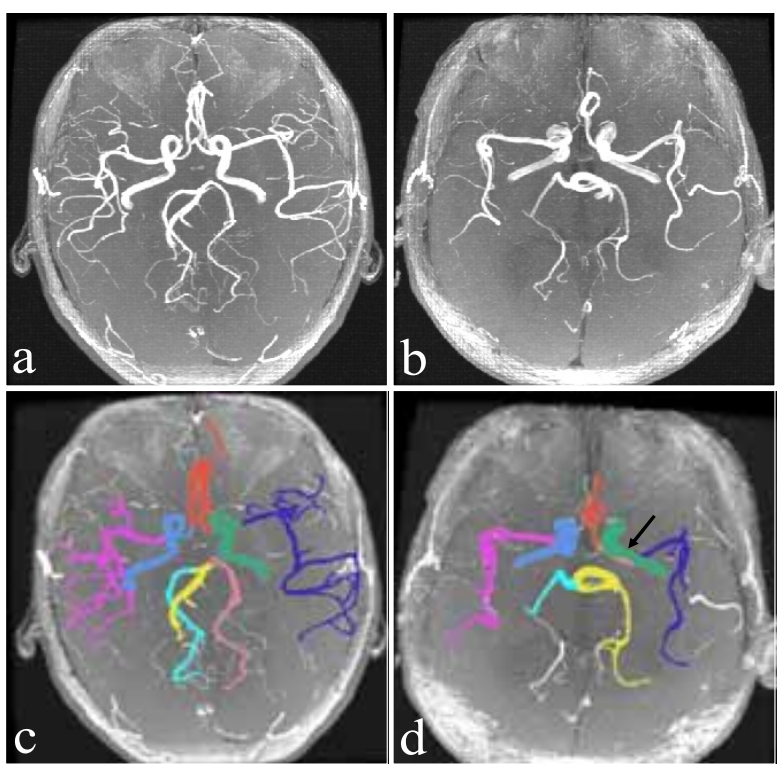

Fig. 5. Example of a 'good' and a 'poor' classification result. (a) and (b) Original MIP images of two different MRA studies. (c) and (d) MIP image as in (a) and (b), respectively, with the classified arteries indicated in different colors. All cerebral arteries in (c) were classified correctly while a part of the cerebral arteries in (d), indicated by a black arrow, was misclassified.

SelMIP image, values of all voxels in the vessel regions other than the selected vessels are set to zero. Fig.6 shows an example of a SelMIP image. Fig.6 (a) and (b) indicate the conventional axial and sagittal MIP images, respectively. As shown in Fig.6 (b), it is difficult to detect small aneurysms in the conventional sagittal MIP image because of the overlapping of the vessels. Figs.6 (c) shows the MIP image in (b) with the classified arteries shown in different colors. Fig. 6 (d) shows the SelMIP image with only one artery (right MCA) selected and displayed. By using the SelMIP image, it is easy to identify small aneurysms. In addition, SelMIP images of views other than the standard sagittal, coronal and axial views can be generated. The additional views can be useful to radiologists in closer examination of the vessels and/or aneurysm candidates in difference viewing directions.

\section{CONCLUSION}

We developed a new viewing technique for the detection of small aneurysms based on the SelMIP images. By using the automated cerebral arteries recognition algorithm presented in this paper, it is easy to make the new SelMIP image consisting of the interested vessels only, rather than all vessels, by manually selecting a cerebral artery from a list of eight. With the new SelMIP image, radiologists can also observe the interested vessels from various directions, visualizing the vessels and aneurysms from views other than the standard sagittal, coronal and axial views. The proposed new viewing technique would be useful in assisting radiologists in detecting aneurysms and in reducing interpretation time.

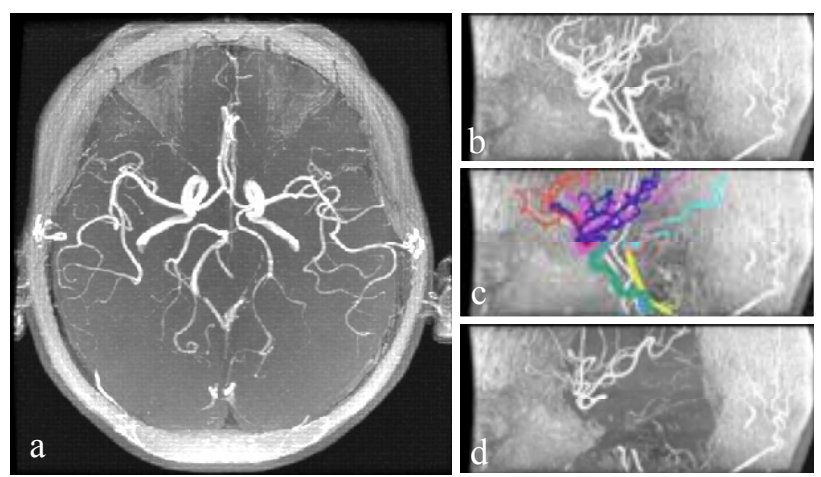

Fig. 6. The SelMIP image. (a) A conventional axial MIP image. (b) The corresponding conventional sagittal MIP image. Identification of individual arteries in this MIP image is difficult. (c) The conventional sagittal MIP image with the classified arteries indicated in different colors. (d) The SelMIP image with the selected right MCA only.

\section{ACKNOWLEDGMENT}

The authors thank Hitoshi Futamura, Akiko Kano, Ph.D., and Hitoshi Yoshimura of Konica Minolta Medical \& Graphic, Inc. and Yoshinori Hayashi and Masakatsu Kakogawa of TAK CO. Ltd. for their valuable suggestions. The authors also thank to Gobert Lee for editing manuscript and to the members of Fujita Laboratory in the Department of Intelligent Image Information, Graduate School of Medicine, Gifu University.

\section{REFERENCES}

[1] Health and Welfare Statistics Association, "Vital Statistics of Japan 2003," volume 1, 300-301, 2003

[2] J. M. Wardlaw and P. M. White, "The detection and management of unruptured intracranial aneurysms," Brain, 123, 205-221, 2000.

[3] H. Arimura, Q. Li, Y. Korogi, T. Hirai, H.Abe, Y. Yamashita, S Katsuragawa, R. Ikeda, and K. Doi, "Automated computerized scheme for detection of unruptured intracranial aneurysms in three-dimensional MRA,” Academic Radiology, 11(10), 1093-1104, 2004.

[4] H. Arimura, Q. Li, Y. Korogi, T. Hirai, S. Katsuragawa, Y. Yamashita, K. Tsuchiya, and K. Doi, "Computerized detection of intracranial aneurysms for three-dimensional MR angiography: Feature extraction of small protrusions based on a shape-based difference image technique," Medical Physics, 33(2), 394-401, 2006.

[5] Y. Uchiyama, H. Ando, R. Yokoayama, T. Hara, H. Fujita, and T. Iwama, "Computer-Aided Diagnosis Scheme for Detection of Unruptured Intracranial Aneurysms in MR Angiography," Proc of the 2005 IEEE Engineering in Medicine and Biology 27th Annual Conference Shanghai, China, paper\#296, 2005.

[6] A. A. Goshtasby, "2-D and 3-D image registration," John Wiley \& Sons, Inc., Hoboken, New Jersey, 2005.

[7] K. S. Arum, T. S. Huang, and S. D. Blostein, "Least-square fitting of two 3-D point sets," IEEE Trans. on Pattern Analysis and Machine Intelligence, 9(5), 698-700, 1987.

[8] P.J. Besl and N. D. McKay, "A method for registration of 3-D shapes," IEEE Trans. on Pattern Analysis and Machine Intelligence, 14(2), 239-256, 1992.

[9] J. Feldmar, J. Declerck, G. Malandain, and N. Ayache, "Extension of the ICP algorithm to nonrigid intensity-based registration of 3-D volumes," Computer Vision and Image Understanding, 66(2), 193-206, 1997. 